\title{
Eutanasia y acto médico
}

\author{
GRUPO DE ESTUDIOS DE ÉTICA CLÍNICA DE LA \\ SOCIEDAD MÉDICA DE SANTIAGO*
}

El presente trabajo no cuenta con Grants ni financiamiento externo.

Recibido el 26 de enero de 2011, aceptado el 29 de abril de 2011 .

Correspondencia a:

Dr. Carlos Echeverría Bunster

Hospital Naval "Almirante Nef"

Subida Alessandri s/n Viña del Mar.

Teléfono: 032-2573380

E-mail: ciecheverria@ hotmail.com

\section{Euthanasia and medical act}

Right to life -as the prohibition of intentionally and arbitrarily taking life, even with authorization of the concerned one- is an internationally recognized right. In many countries, debate regarding euthanasia is more centered in its convenience, social acceptability and how it is regulated, than in its substantial legitimacy. Some argue that euthanasia should be included as part of clinical practice of health professionals, grounded on individual's autonomy claims-everyone having the liberty to choose how to live and how to die. Against this, others sustain that life has a higher value than autonomy, exercising autonomy without respecting the right to life would become a serious moral and social problem. Likewise, euthanasia supporters sometimes claim a 'right to live with dignity', which must be understood as a personal obligation, referred more to the ethical than to the strictly legal sphere. In countries where it is already legalized, euthanasia practice has extended to cases where it is not the patient who requests this but the family or some healthcare professional, or even the legal system-when they think that the patient is living in a condition which is not worthy to live. Generalization of euthanasia possibly will end in affecting those who need more care, such as elder, chronically ill or dying people, damaging severely personal basic rights. Nature, purpose and tradition of medicine rule out the practice of euthanasia, which ought not be considered a medical act or legitimately compulsory for physicians. Today's medicine counts with effective treatments for pain and suffering, such as palliative care, including sedative therapy, which best preserves person's dignity and keeps safe the ethos of the medical profession.

(Rev Med Chile 2011; 139: 642-654).

Key words: Bioethical Issues; Codes of Ethics; Ethics, medical; Euthanasia; Terminal Care.
E n publicaciones anteriores nuestro grupo se ha referido a diversos temas relacionados con el final de la vida ${ }^{1-4}$, lo que guarda relación con la eutanasia, objeto de este trabajo. Aún cuando la solicitud de eutanasia es excepcional en nuestro medio, parece necesario reflexionar desde la profesión médica acerca de una materia que incide fuertemente sobre el sentido y la práctica de la misma.

En la actualidad la discusión sobre la eutanasia ha alcanzado connotación pública, lo que parece obedecer a diversos factores. El desarrollo tecnoló-

\footnotetext{
*Documento elaborado por el Grupo de Estudios de Ética Clínica de la Sociedad Médica de Santiago y aprobado por su Directorio (8 de marzo de 2011) y Mesa Directiva (29 de abril de 2011). Integrantes del Grupo: Dres. Carlos Echeverría B. (Hospital Naval "Almirante Nef", Viña del Mar), Alejandro Goic G. (Academia de Medicina del Instituto de Chile), Carolina Herrera C. (Facultad de Medicina, Universidad Andrés Bello), Carlos Quintana V. (Facultad de Medicina, Pontificia Universidad Católica de Chile), Alberto Rojas O. (Facultad de Medicina, Universidad Andrés Bello), Rodrigo Salinas R. (Facultad de Medicina, Universidad de Chile), Alejandro Serani M. (Facultad de Medicina, Universidad de Los Andes), Paulina Taboada R. (Centro de Bioética, Pontificia Universidad Católica de Chile), Ricardo Vacarezza Y. (Presidente del Grupo de Estudios; Facultad de Medicina, Universidad de Chile). (Una versión extendida de este documento recibió en diciembre de 2010 el Premio de Ética del Colegio Médico de Chile).
} 
gico ha posibilitado la recuperación de enfermos y ha permitido prolongar la vida de otros, pero con elevados costos personales, económicos y sociales. El uso indiscriminado de estas tecnologías ha conducido también al llamado "ensañamiento terapéutico" $\mathrm{u}$ "obstinación terapéutica", que, por tratar de conservar la vida a toda costa, llega a ser desmesurado ${ }^{7}$.

La preocupación por la eutanasia está además relacionada con el envejecimiento de la población. En Chile, la expectativa de vida al nacer ha pasado de 54,8 años en el quinquenio $1950-55$, a 74,8 años en el 2000-05 $(+42 \%)^{8}$. Se proyecta que los mayores de 65 años representarán $11 \%$ en el 2020 y $15 \%$ en el 2030. A su vez el envejecimiento de la población implica un incremento de los costos de la atención de salud, por lo que ha surgido la pregunta económica si acaso la sociedad debiera restringir la atención médica de los ancianos en la etapa final de su vida9.

Los resultados de las encuestas de opinión son difíciles de interpretar y no permiten discriminar entre la voluntad de legislar sobre la eutanasia y la de aplicarla realmente a seres queridos ${ }^{10}$. Una encuesta, realizada en la ciudad de Concepción, muestra que $32,7 \%$ de quienes responden cree que la eutanasia se debe regular legalmente, a la vez que $63,8 \%$ no la aplicaría al hijo, padre o hermano ${ }^{11}$. Estos resultados sugieren una confusión entre el significado preciso de eutanasia y de otros conceptos relacionados con ella, tales como la limitación del esfuerzo terapéutico y la obstinación terapéutica.

Finalmente, en el trasfondo de la discusión sobre eutanasia, están en juego temas valóricos como el respeto de la vida humana, la autonomía individual y la responsabilidad propia y ajena en la toma de decisiones.

\section{Sobre el término eutanasia y su concepto}

La idea que la muerte puede ofrecer un alivio para una vida condenada al sufrimiento ya aparece en el mito del Centauro Quirón, quien gravemente herido, y sin poder curar ni morir, solicita a Apolo que, por gracia, le otorgue la muerte para terminar con su tormento ${ }^{12}$.

La palabra 'eutanasia' (del gr. $\varepsilon U \tilde{,}$, bien, y $\theta$ áv $\alpha \tau$ ○s, muerte) ha tenido diversos significados. Según Suetonio, el emperador César Augusto la habría utilizado para indicar su anhelo de tener una muerte natural rápida y $\sin$ dolor $^{13}$. La ambigüedad en el uso de la palabra eutanasia suele dificultar la discusión del tema. En ocasiones, se ha denominado eutanasia a actos que, en rigor, corresponderían más bien a acciones eugenésicas, suicidas u homicidas. Este fue el caso en la Alemania nazi ${ }^{14}$, en que se dio este nombre a la "muerte por piedad (Gnadentodt)" aplicada a aquellos individuos cuyas vidas se estimaban indignas de ser vividas ${ }^{15}$.

La Real Academia de la Lengua Española ${ }^{16}$ define la eutanasia como "acción u omisión que, para evitar sufrimientos a los pacientes desahuciados, acelera su muerte, con su consentimiento o sin él"; o, como "muerte sin sufrimiento físico". De un modo más técnico, también se la ha definido como "la muerte indolora infligida a una persona humana, consciente o no, que sufre abundantemente a causa de enfermedades graves e incurables o por su condición de disminuido, sean estas dolencias congénitas o adquiridas, llevada a cabo de manera deliberada por el personal sanitario o al menos con su ayuda, mediante fármacos o con la suspensión de curas vitales ordinarias, porque se considera irracional que prosiga una vida que, en tales condiciones, se valora como ya no digna de ser vivida" ${ }^{17}$. Por otra parte, la Declaración 'Iura et Bona' de la Iglesia Católica sobre la eutanasia la define como "una acción o una omisión que por su naturaleza, o en la intención, causa la muerte, con el fin de eliminar cualquier dolor"18.

En otro sentido, se han aplicado diversos adjetivos a la noción de eutanasia. Se ha distinguido, por ejemplo, la eutanasia directa de la indirecta, aludiendo esta última a la supresión del dolor con fármacos que pueden contribuir a acortar la vida ${ }^{19}$. Otros autore ${ }^{20}$ diferencian la eutanasia 'activa' de la 'pasiva': Es 'activa' “cuando la muerte se provoca por medio de una acción, como pudiera ser la administración de dosis mortales de estupefacientes o de una substancia letal"; y 'pasiva', cuando "la muerte es consecuencia de la omisión, o de la interrupción, de intervenciones cuyo objetivo es simplemente el de ofrecer a la vida su soporte indispensable (nutrición, hidratación, etc.)”. Más específicamente, la eutanasia activa se ha definido como "la acción que tiene por objeto acabar deliberadamente con la vida de una persona que padece una enfermedad irreversible, sufrimientos intolerables y que lo pide de forma autónoma y reiterada"21. 
En Holanda, en abril de 2002, se aprobó que la eutanasia y el suicidio asistido son legales si se cumplen los siguientes requisitos: que el paciente lo requiera de modo voluntario y lo haya considerado adecuadamente; que su condición sea intolerable y sin esperanzas; que no hayan disponibles alternativas aceptables de tratamiento; que el método utilizado sea médica y técnicamente apropiado, y que se consulte a otro médico antes de proceder. Dados estos requisitos, la ley obliga a reportar esta muerte como no natural para que esta acción sea considerada legal ${ }^{22}$.

En síntesis, en el uso actual del término, podrían reconocerse las siguientes condiciones, que debieran ser copulativas:

a) presencia en determinada persona de una enfermedad incurable, que le provoca un dolor o sufrimiento que ella, u otro, considera intolerable;

b) ejecución de un acto humano deliberado, cuyo objeto es acabar con la vida de otra persona; y

c) motivación originada sea en la compasión por el otro, o en el valor que se le adjudica a esa vida en términos de costo-beneficio, no justificando el que ella sea vivida.

Todo lo anterior previa voluntad expresa del sujeto o de su representante. Sin embargo, existe una controversia sobre si es necesario que éste lo solicite explícitamente ${ }^{23}$.

En términos éticos y jurídicos habrá que distinguir también situaciones diferentes como mala práctica, negligencia en las acciones o en el otorgamiento de tratamientos acordes con el desarrollo de la ciencia y la lex artis; homicidio propiamente tal según las circunstancias señaladas por el Código Penal $^{24}$; o bien, limitación razonada del esfuerzo terapéutico al evitar tratamientos extraordinarios desproporcionados, respetando la dignidad de la persona y el proceso natural de la muerte ${ }^{25}$.

Tampoco se debiera confundir con eutanasia el hecho de utilizar medicamentos para aliviar el dolor, sin intención de causar la muerte, aún cuando ello pueda originar un acortamiento de la vida del paciente; es la llamada "acción de doble efecto" 26 , también denominada del "voluntario indirecto", que entiende que no se puede optar directamente por el mal y que no es éticamente apropiado alcanzar un buen fin mediante acciones malas, siendo esencial en esta consideración el objeto directo del acto. Quienes discuten el uso de este criterio moral arguyen que la intencionalidad es difícil de objetivar ${ }^{27}$. Se ha aducido, también, que este criterio corresponde a una tradición religiosa particular; que es difícil de validar externamente; que las intenciones no están sujetas a la ley, como sí lo están las consecuencias previsibles y que, adicionalmente, contraría la autonomía personal ${ }^{28}$. Finalmente, resulta también necesario considerar los eventuales abusos de este principio, el cual no debe ser utilizado como una "excusa multifuncional que sirva para introducir cualquier tipo de excepción a la estricta protección de los bienes humanos básicos"29.

\section{Desafíos que se plantean hoy a la medicina}

Precisado el significado del término eutanasia, conviene ahora examinar algunas situaciones concretas, surgidas desde la medicina, que han favorecido la aparición de demandas sociales en pro de legitimar las acciones eutanásicas.

El dolor físico y el sufrimiento concomitante podrían llegar a ser subjetivamente intolerables para quien lo padece. Si bien el paciente solicita que tal dolor o sufrimiento desaparezca, se atenúe o vuelva soportable, algunas veces la muerte pudiese visualizarse como una solución definitiva al problema.

El desarrollo de la investigación médica y la tecnología, con su positivo aporte tanto al diagnóstico como a la terapia de las enfermedades, ha traído anexo un incremento de los costos de la atención en salud, tanto para prestadores como beneficiarios. Adicionalmente, la introducción de intereses relacionados con la medicina empresarial, incluyendo la intermediación de prestaciones sanitarias por entes financieros, ha contribuido a acrecentar los costos globales de la medicina. Un criterio de balance costos/beneficios centrado en la disminución o ausencia de productividad de pacientes aquejados de ciertas enfermedades, versus el volumen de recursos orientados a su atención, podría estimar desproporcionado, en términos económicos, atender a personas que en definitiva van a morir en un plazo breve. Acelerar la muerte de esos individuos podría entenderse como una forma de optimizar la utilización de los recursos involucrados. Siguiendo esta lógica, se ha llegado a plantear, incluso, el "deber de morir" 30 .

Por otra parte, los cambios estructurales de 
la familia y de las características de las viviendas, han hecho disminuir los espacios y condiciones destinados a mantener y cuidar los pacientes crónicos incapacitados. Estos elementos, junto con el incremento del costo de la vida, han conducido a que, quienes carecen del sostén de su familia más inmediata, queden sujetos sólo al apoyo que les puedan brindar los sistemas de salud, especialmente en el ámbito hospitalario. Ello hace recaer sobre el sistema sanitario y su personal el peso de la falta de soporte social. Ocasionalmente los propios enfermos expresan su temor al abandono afectivo y social, temiendo ser una carga excesiva para los suyos, viendo con desesperanza que sólo la muerte podría liberarlos de una condición penosa. Ante un paciente terminal en el que, junto a sus molestias y costos personales, se añaden los de la familia ${ }^{31}$ y/o del sistema de salud, algunos se preguntan si no sería mejor poner término a la situación acelerando su deceso.

Hay pacientes no terminales aquejados de enfermedades que, en el mediano plazo, tienen un mal pronóstico o son progresivas, sin cura posible en la actualidad y con probabilidad significativa de evolucionar a un deterioro grave de la condición física o mental. Ello implica un compromiso de su calidad de vida, que perciben como un menoscabo de su dignidad personal. Las penurias que, en estos casos, se le impone a la familia y/o la sociedad, son también invocadas por algunos como razón suficiente para solicitar la muerte de esos individuos.

El aumento de la prevalencia de enfermedades crónicas invalidantes, concomitante con la situación demográfica actual, configurará un grupo de personas mayores que, en el último año de su vida, podrían llegar a consumir mucho más dinero en tratamiento de enfermedades respecto al que aportaron durante su vida laboral a los sistemas previsionales. En los Estados Unidos de América, las estimaciones desarrolladas concluyen que "el costo de proveer cuidados de salud para un anciano es de tres a cinco veces mayor que el de alguien menor de 65 años. De ello resulta, como proyección para el año 2030, que el gasto de la nación en cuidados de salud se incrementará en 25\% a causa de estos cambios demográficos ${ }^{32}$ ". Por estas razones ha surgido incluso la idea de optimizar la utilización de los recursos sanitarios, acelerando el fallecimiento de aquellos pacientes crónicos incurables que reciben cuidados paliativos ${ }^{33}$ o de los ancianos con enfermedades crónicas invalidantes.
Por su parte, el desarrollo de las tecnologías asociadas a la Medicina ha tendido a generar expectativas ilusorias con respecto a las posibilidades de la recuperación de la salud. A ello se suma la publicidad que -en ocasiones- exagera el valor de determinadas terapias $u$ ofrece expectativas inalcanzables. El desengaño con respecto a sus reales resultados conduce a frustraciones que pudieran llevar a solicitar una intervención para procurar la muerte.

Dada la creciente conciencia contemporánea sobre el valor de la autonomía personal, cada vez son más numerosas las personas que invocan el 'derecho' a terminar con su vida del modo más eficaz y menos molesto. Para ello se apela a los médicos y la Medicina para que utilicen sus conocimientos y recursos con el fin de acabar con la vida de quien libremente lo solicite.

En otro sentido, la ausencia de acciones terapéuticas o correctivas en pacientes portadores de anomalías genéticas y/o malformaciones que condicionan grave incapacidad, ha llevado a algunos a considerar la supresión de esas vidas estimadas -generalmente por terceros- como de pésima calidad ${ }^{34,35}$.

Debe considerarse, además, que con frecuencia se habla de "muerte digna" o del "derecho a morir con dignidad", sin explicitar claramente que se entiende por ello. Así, el espectro es amplio, desde quienes invocan como fundamento la autonomía personal, sosteniendo que la vida no es un deber sino un derecho renunciable, hasta aquellos que hacen ver la falta de sensatez de los tratamientos desproporcionados, manifestándose en consecuencia, visiones diferentes sobre el sentido del sufrimiento ${ }^{36}$.

\section{¿Debe la medicina responder a estos desafíos?}

Frente a la diversidad de argumentos y situaciones que se esgrimen para justificar las demandas actuales de una liberalización, e incluso de la institucionalización de la eutanasia, se hace necesario reflexionar sobre la obligación que tiene la medicina de dar una respuesta, dando cuenta de la naturaleza y eficacia de las soluciones médicas para enfrentar algunas situaciones clínicas complejas. Para ello consideraremos, de modo crítico, como se plantea la profesión médica frente a cada uno de los desafíos enumerados anteriormente, enten- 
diendo por soluciones 'médicas' sólo aquellas que responden a la definición y fines de la medicina.

Acorde con lo indicado en el "Diccionario de la Lengua Española"16, se entiende por Medicina a la "ciencia y arte de precaver y curar las enfermedades del cuerpo humano". Entre los anglosajones, Dorland ${ }^{37}$ define la medicina como "el arte y la ciencia del diagnóstico y el tratamiento de la enfermedad y la conservación de la salud"; también se la ha descrito como "la ciencia y arte de diagnosticar, tratar, curar $y$ prevenir la enfermedad, aliviar el dolor y mejorar y preservar la salud" 38 , o como "la ciencia o práctica del diagnóstico y el tratamiento de enfermedades y lesiones y la preservación de la salud"39. El "Petit Robert" la define como la "ciencia que tiene por objeto la conservación y el restablecimiento de la salud"40.

Este somero examen muestra como en el lenguaje común se contienen y expresan los fines de la medicina. Por ello, no es de suyo evidente hasta qué punto sea competencia de consensos ideológicos o políticos circunstanciales modificar estos fines, de profundas raíces antropológicas.

\section{El dolor y su manejo}

Actualmente, se dispone de conocimientos avanzados sobre la fisiología, psicología y manejo del dolor ${ }^{41}$, tanto en su forma aguda como crónica, junto con medicamentos, que -apropiadamente utilizados- pueden surtir efectos beneficiosos en los pacientes que los requieren. Diversos centros internacionales han hecho aportes en este campo y en Chile existe una Guía Clínica para el manejo del dolor secundario al cáncer avanzado, elaborada por iniciativa del Ministerio de Salud ${ }^{42}$.

En el ámbito de los cuidados paliativos, se han desarrollado métodos para el manejo de los llamados "síntomas refractarios" ${ }^{43}$ : síntomas que no pueden ser controlados adecuadamente a pesar de esfuerzos agresivos para lograr un tratamiento tolerable que no comprometa la conciencia del paciente. Para estos casos se han preconizado diversas formas de la denominada "sedación paliativa" 44 o "sedación terminal". La Sociedad Española de Cuidados Paliativos advierte sobre la posible confusión a que puede dar lugar este último término, "ya que puede interpretarse como que es la sedación la que termina con el paciente" 45 , debiendo también considerarse los eventuales abusos que se pueden cometer con el empleo de esta metodología. Por todo ello, hoy se tiende a hablar de "sedación terapéutica" 46 que, en el con- texto de los cuidados paliativos, se define como el uso monitorizado de medicamentos orientados a inducir una disminución o ausencia del estado de alerta, con el objeto de aliviar un sufrimiento, intratable de otro modo, en una forma éticamente aceptable para el paciente, su familia y los proveedores de cuidado sanitario. Debemos reconocer que no todos los profesionales de la salud están -de hecho- suficientemente al corriente de estos avances, o bien carecen del entrenamiento práctico requerido. Adicionalmente se hace necesario desarrollar otros mecanismos seguros para una mejor disponibilidad de ciertos analgésicos, cuyo uso es restringido debido a los eventuales abusos que deben ser prevenidos por la autoridad.

En suma, nos parece claro que, con un mayor desarrollo en el campo de los cuidados paliativos, un conocimiento más extensivo, un mejor entrenamiento y adecuada reglamentación, la profesión médica podría y debería contribuir a modificar la imagen pública de temor e indefensión frente al dolor.

\section{Incremento de los costos de la atención de salud}

Aun cuando el progreso científico y técnico de la medicina, con su consiguiente aumento de los costos, ha permitido grandes logros en la atención de salud, es importante reconocer que no siempre existe una relación directa entre mayor tecnología y mejor calidad de la medicina. En consecuencia el mero hecho que una tecnología esté disponible no implica necesariamente que deba ser utilizada. Es un desafío original, impuesto a la medicina actual por el desarrollo científico y tecnológico, buscar y descubrir lo que técnica y éticamente se debe llevar a cabo en el manejo de un paciente específico, sin que los costos se hagan inabordables. El saber y el arte médicos se encuentran empeñados en concurrir a este desafío.

Es tarea de quienes asignan y administran los recursos disponibles para la salud distribuirlos de un modo justo, propendiendo a un uso óptimo de ellos. También la sociedad debiera debatir y, a través de las organizaciones pertinentes, definir qué cantidad de recursos quiere utilizar en salud, asumiendo las consecuencias de sus decisiones, sin hacer recaer sobre el personal sanitario la responsabilidad de asignaciones insuficientes para las verdaderas necesidades de los pacientes. Los médicos debiesen contribuir con su saber a esta discusión, a la vez que ser diligentes en el uso de los recursos 
asignados. Un debate social abierto y responsable debiera contribuir a ajustar las expectativas de la población a las posibilidades reales de satisfacción de las necesidades. Hay que reconocer, con pesar, que este debate es todavía escaso en nuestro medio.

Dada la multiplicidad de factores biológicos, psicológicos, ambientales y sociales que influyen en la salud ${ }^{47}$, es preciso tomar en consideración cada uno de estos componentes. Para enfatizar la necesidad de involucrar a toda la comunidad (tanto a nivel personal como colectivo) en la reducción de los costos de la atención de salud baste mencionar, por ejemplo, los efectos beneficiosos de los programas de vida saludable y actitudes preventivas para la Diabetes Mellitus ${ }^{48}$, como también lo que sucede con el tabaco, el alcohol y las drogas de abuso, tanto en su consumo como con la abstención de ello. Las carencias en las acciones preventivas eficaces se traducen en el aumento de enfermedades graves y consumidoras de recursos, impidiendo que éstos sean redistribuidos a aquellas áreas donde todavía no se cuenta con medidas de prevención.

La optimización del uso de los recursos para la salud puede contribuir significativamente a disminuir o contener los costos de las prestaciones de salud, sin necesariamente rebajar su calidad. Lo anterior supone consensos sociales y voluntades políticas que van mucho más allá del alcance de la profesión médica.

\section{Carga para la familia o sociedad}

Este concepto, de clara connotación económica, tiene también un componente fundamental relativo al respeto y solidaridad hacia las personas.

El aporte de la medicina en el cuidado de los enfermos crónicos o desvalidos, consiste primariamente en su adecuada acogida en los servicios asistenciales, por razones médicas. Las soluciones de fondo a la atención humana de estas personas es tarea de la sociedad en su conjunto, a través de la seguridad social y el sostén familiar del individuo. Los sistemas de seguridad social debieran financiar los subsidios que se requieran para las personas de menores recursos, como también quienes poseen medios para ello debieran hacerse cargo de las responsabilidades que les son propias.

En el caso de enfermos que sufren de abandono afectivo o social, y en consonancia con lo anteriormente expresado, los cambios observados en la estructura de la familia y de la sociedad escapan al ámbito y responsabilidad directa de la Medicina. No parece entonces lógico pedirle a ella la solución a este problema, ni menos aún, con este pretexto, proponerle poner término a la vida de personas vulnerables, cuyo apoyo debe resolverse por otras vías, sean ellas la educación, la seguridad social, la estructura impositiva u otras que se diseñen. Hacemos presente, también, lo difícil que resulta evaluar la calidad de vida de una persona y el subjetivismo que ello involucra, tanto desde el punto de vista del paciente como del médico tratante ${ }^{4}$.

\section{Visualización de un límite temporal a la acción terapéutica}

Tal es el caso de los pacientes terminales ${ }^{1}$. En las últimas décadas se ha desarrollado un movimiento que ha planteado la utilidad de los cuidados paliativos para la atención de estos enfermos. Desde 1967, en el St. Christopher Hospice ${ }^{49}$, y a partir de los esfuerzos de Cicely Saunders, se persiguen los objetivos de aliviar el dolor y el sufrimiento a los pacientes terminales, hacer posible una "buena" muerte y ayudar a la familia en este trance y asistir en la búsqueda de significado de la situación ${ }^{50}$. Lo característico de esta instancia es el foco en la persona como ser integral (físico, psicológico, social y espiritual), centrarse en el paciente, la apertura y honestidad en la comunicación y la aceptación de la inevitabilidad de la muerte, ligado todo a un mejoramiento de la calidad de vida del paciente, mediante un trabajo multiprofesional integrado con voluntarios de la comunidad ${ }^{51}$. Este enfoque y sus variantes, conducen a una respuesta que acoge al paciente en la etapa final de su vida, con actitudes positivas e integradoras, respetuosas de la dignidad personal. La generalización de este tipo de actitudes y destrezas a la globalidad de los profesionales de la salud debiese ser un objetivo a lograr, no sólo por parte de la profesión médica, sino de la sociedad en su conjunto. El examen serio de los obstáculos que se oponen a este objetivo, así como la búsqueda de soluciones convenientes, aceptables y factibles, debiese ser también una meta en este campo.

\section{Cambios demográficos}

$\mathrm{Al}$ respecto, se debe considerar lo señalado por Marín y colaboradores ${ }^{52}$, en el sentido de que casi la mitad de la población nonagenaria se mantiene en condiciones cognitivas, de desplazamiento y autonomía personal aceptables. Por ello se sostie- 
ne que es factible y necesario un enfrentamiento activo del problema por el equipo de salud, con un enfoque individualizado, multidisciplinario y global, que intervenga en la prevención, manejo y rehabilitación de la discapacidad, con el fin de mejorar la calidad de vida de esos adultos mayores. Esta es una respuesta que surge desde la medicina, con herramientas propias y sin vulnerar la vida de estas personas.

\section{Expectativas irreales en las capacidades tecnológicas de la medicina}

Es posible que ellas se generen desde un sesgo o falta de información objetiva sobre las verdaderas y reales capacidades de la medicina, como ciencia y como técnica. Entregar información veraz y oportuna corresponde al personal de salud, lo que debe incluir el reconocimiento de los límites de la medicina. Sin embargo, los medios masivos de comunicación y la publicidad médica inadecuada suelen confundir a la opinión pública, cuando atienden solamente a aquellos aspectos espectaculares de la noticia, o muestran como habituales situaciones que son de excepción. Cabe señalar que la presentación adecuada y veraz de la información correcta entregada por el médico, corresponde al emisor o difusor, quien debe asumir su papel y propia responsabilidad. Además, la irrupción de una lógica del mercado, orientada principalmente al consumo, en la promoción de las atenciones relacionadas con la medicina, ha introducido en los últimos años un nuevo elemento perturbador que tiende a distorsionar el valor objetivo de las acciones de salud.

\section{Autonomía personal y sus límites}

La autonomía, valor eminente de la vida personal, tiene sus limitaciones, como es propio en toda realidad humana. Las condiciones extremas en que se suele plantear la eutanasia en el ámbito médico hacen, efectivamente, poner en duda la libertad con que esta se solicita. $\mathrm{Al}$ respecto cabe mencionar a Kant: "no hay, en rigor, moralidad si no hay independencia respecto a cualquier objeto deseado" ${ }^{53}$.

Por otra parte, la alta valoración del respeto debido a la autonomía de los pacientes, en la época contemporánea, no parece haber ido a la par con el reconocimiento del respeto que la sociedad le debe a la autonomía de la profesión médica. Dada la complejidad de los elementos que intervienen en la discusión actual sobre la eutanasia se hace necesario, entonces, determinar, desde la profesión médica, cual sea su ámbito propio de autonomía, con el objeto de evitar solicitudes fuera de su competencia y/o contradictorias con la naturaleza y tradición de la medicina.

\section{Consideraciones éticas}

\section{Antecedentes históricos}

En la antigüedad griega y romana, eutanasia designaba más bien un deber del médico, consistente en proporcionarle al enfermo una 'buena muerte' por medio de los cuidados adecuados ${ }^{54}$. En ese mundo greco-romano era común la práctica de eliminar niños malformados ${ }^{55} \mathrm{y}$ el suicidio y el aborto eran aceptados en la mayoría de las ciudades griegas. También en algunas épocas y culturas, los ancianos eran abandonados a su propia suerte ${ }^{56}$.

En el siglo V a.C. la Escuela Hipocrática adoptó una posición radicalmente diferente a la que prevalecía en su época, condenando explícitamente el aborto y la eutanasia. Es así como, el Juramento Hipocrático señala: "No daré a nadie, aunque me lo pida, ningún fármaco mortal, ni haré semejante sugerencia. Igualmente no proporcionaré a mujer alguna un pesario abortivo. En pureza y santidad mantendré mi vida y mi arte" 57 .

En los siglos posteriores a Hipócrates, esta normativa médico-deontológica fue asimilada en diversas culturas. Así, la implícita o explícita prohibición al médico de practicar la eutanasia aparece también en diversos códigos contemporáneos de Ética Médica.

Un estudio contemporáneo sobre Códigos de Ética y Deontología Médica de 39 países ${ }^{58}$, mostró que las discusiones actuales, contrariamente a lo ocurrido en la opinión pública, han conducido, entre los médicos, a una cada vez más clara prohibición de recurrir a la eutanasia y el suicidio asistido. Así, la Asociación Médica Mundial, en 1987, establece que la eutanasia es contraria a la ética; en la Asamblea Mundial de 1992 expresa su oposición al suicidio médicamente asistido; $y$, en su resolución de 2002, reafirma "su firme convicción de que la eutanasia entra en conflicto con los principios básicos de la ética médica"59.

Finalmente, varios documentos, en el ámbito internacional $^{60-63}$, han hecho expresa mención del 
respeto inherente a la vida humana y la dignidad de las personas, implicando explícita condena a las violaciones de estos derechos humanos.

Actualmente, y pese a la opinión contraria de instituciones médicas internacionales reconocidas, la eutanasia ha sido legalizada en algunos países, como por ejemplo, en Holanda ${ }^{64}$ (2002) y en Bélgica $^{65}$ (2002).

En Chile, el artículo $9^{\circ}$ del Código de Ética del Colegio Médico ${ }^{66}$, señala que: "El médico no podrá realizar acciones cuyo objetivo directo sea poner fin a la vida de un paciente bajo consideración alguna", quedando claro el rechazo a la eutanasia. El Código Penal chileno, en su título VII ${ }^{67}$, castiga "al que mate a otro" con penas aflictivas. Adicionalmente, en el artículo 393, del mismo título, castiga a quien "con conocimiento de causa prestare a otro auxilio para que se suicide".

\section{Los fines de la Medicina}

La valoración ética de la eutanasia puede examinarse en referencia a la consideración de los fines de la medicina. Goic ${ }^{68}$ ha señalado que "la medicina persigue un bien -ayudar al enfermo$y$, por lo tanto, tiene un objetivo ético; en otras palabras, en su esencia, es de naturaleza ética y asistencial"; como también recuerda que "la muerte inducida para poner fin al sufrimiento de un paciente desahuciado es una acción reñida con el propósito fundamental de la disciplina médica".

En el Hastings Center, centro relevante de la discusión bioética en los EE.UU., se desarrolló un informe, acorde con el cual ${ }^{69}$ los fines de la medicina serían los siguientes:

i. La prevención de enfermedades y lesiones, y la promoción y la conservación de la salud;

ii. El alivio del dolor y el sufrimiento causados por males;

iii. La atención y la curación de los enfermos y los cuidados a los incurables;

iv. La evitación de la muerte prematura y la búsqueda de una muerte tranquila.

El segundo y el último pudieran relacionarse con la eutanasia. Ahora bien, para el alivio del dolor y el sufrimiento -reconociendo que se trata de dos conceptos diferentes- existen soluciones de tipo farmacológico u otras (algunas de ellas fuera del ámbito de la medicina) para intentar resolverlos o, al menos, atenuarlos significativamente. Con respecto a la muerte tranquila es pertinente recordar que el informe mencionado señala que "puede definirse como aquella en la que el dolor y el sufrimiento se reduzcan mediante unos cuidados paliativos adecuados, en la que al paciente nunca se le abandone ni descuide y en la que los cuidados se consideren igual de importantes para los que no vayan a sobrevivir como para los que sì'70. Lo anterior implica el debido acompañamiento del paciente por parte de su familia y del personal sanitario, con un trabajo y preocupación mayores, atendiendo a considerarle como un ser desvalido que precisa ayuda y compasión, en vez de recurrir a la simple y expedita solución de eliminarlo. En Chile, si bien se cuenta con una organización y estructura asistencial para el tratamiento de los casos de dolor en cáncer avanzado ${ }^{71}$, hay todavía un amplio campo de desarrollo para los cuidados paliativos.

\section{El acto médico}

Las acciones específicas que lleva a cabo un médico clínico son diagnosticar, tratar y formular el pronóstico de la enfermedad que padece una persona $^{72}$. Además, en su función educadora, aconseja al paciente sobre las medidas preventivas que impidan o aminoren la posibilidad de recaer en su enfermedad. Así, toda acción que emprende el médico para lograr los propósitos señalados es un acto médico.

El acto médico es un acto libre llevado a cabo por un profesional que tiene como campo de responsabilidad la salud del paciente que está bajo su cuidado. Un médico es requerido por un paciente que desea sanar o aliviar y que necesita de su apoyo para recuperar su salud, lo que el médico intentará lograr con los medios a su alcance ${ }^{73}$. En último término, lo que el médico persigue, cuando es posible, es contribuir a recuperar la salud de sus pacientes y a ello ha sido orientada su educación profesional. Es claro que el médico no ha sido formado ni entrenado para poner fin intencionalmente a la vida de sus pacientes, porque esto sería contradictorio con el propósito esencial de la medicina y su tradición. Al respecto, la antropóloga Margaret Mead ha afirmado que históricamente el Juramento Hipocrático constituyó un avance extraordinario, no sólo para la medicina sino para toda la humanidad, al separar, por primera vez, para el médico, la capacidad de sanar de la capacidad de matar; atributos que, en otras sociedades, recaían en una misma persona. 
Por ello señala que quienes dictan las políticas deben proteger este inestimable descubrimiento, que compromete a la profesión médica con el respeto a la vida ${ }^{74}$.

La Eutanasia no persigue diagnosticar, tratar ni prevenir una enfermedad, sino que es una acción ejecutada con el propósito intencionado de causar la muerte de una persona para evitar el dolor y/o sufrimiento, lo que excede la naturaleza y objetivos de un acto médico. Así, el profesional que interviene en la eutanasia no está realizando una acción propia de su profesión, y contradice los fundamentos del saber médico y los valores del Juramento Hipocrático. Lo cierto es que no suele ser el médico sino la familia, el propio paciente o la presión de la sociedad expresada, de diversas maneras, los que plantean la aplicación de la eutanasia.

Quienes favorecen la eutanasia argumentan que, con ella, se estaría cumpliendo uno de los objetivos de la medicina que es aliviar el dolor o el sufrimiento - en este caso extremos- y sería, por consiguiente, un acto de beneficencia. Sin embargo, para juzgar la bondad de una acción se debe considerar no sólo la legitimidad de la intención del agente, sino también la legitimidad de los medios utilizados para llevarla a cabo. En verdad, es difícil sostener que dar término intencionado a la vida de un enfermo, sea un acto que lo beneficie; más bien, parece ser una acción que causa maleficio, ya que no existe un daño mayor que se pueda hacer a una persona que quitarle la vida. En efecto, el valor de la vida humana precede al de la beneficencia, en tanto que la vida es una condición necesaria para poder ejercer aquella: "la vida es el bien fundante, sin ella no podemos gozar de ningún otro bien"75.

Entendiendo que el principio general del respeto a la vida humana no admite excepciones, debemos reconocer la existencia de situaciones límites y de gran dramatismo, en las que sería temerario aventurar juicios categóricos desde el punto de vista moral.

Con respecto a la llamada eutanasia pasiva, es una denominación que más bien dificulta la comprensión del tema. En muchos casos, al hablar de eutanasia pasiva se confunde la eutanasia con una legítima limitación del esfuerzo terapéutico en pacientes terminales. En determinadas condiciones, abstenerse de emplear una tecnología o retirarla si está en uso, es fruto del razonamiento clínico y no una simple omisión; en consecuencia, no es un acto pasivo, sino activo. No hacer nada, para intentar aliviar a un enfermo terminal, no es eutanasia sino una violación positiva del principio de no abandono del enfermo, que obliga profesional y éticamente al médico. Nos parece que la expresión eutanasia pasiva debería eliminarse por equívoca y no contribuir a esclarecer un problema complejo como es la eutanasia. Por cierto, tampoco debe confundirse la eutanasia con el error, la negligencia médica o el homicidio.

\section{La situación en Holanda}

Desde 1994, año en que se establecieron las condiciones para no iniciar una causa legal contra un médico que realizaba la eutanasia, hasta el año 2002, año en que se establece que la eutanasia y el suicidio asistido son legales, Vega y Ortega ${ }^{76}$, contrariamente a van der Heide y cols $^{64}$, han podido demostrar un incremento sostenido de muertes intencionales en el contexto médico.

Por otra parte, aún cuando sólo puede solicitar la eutanasia una persona competente mayor de 16 años, diversas consideraciones han conducido en la práctica a terminar con la vida de recién nacidos. Es así como un grupo de médicos y funcionarios judiciales desarrollaron el Protocolo Groningen ${ }^{34}$, que establece requisitos para la eutanasia en recién nacidos, soslayando con medidas administrativas el mandato legal.

Los datos señalados permiten concluir que en la situación holandesa se ha llegado a una legitimación jurídica de una conducta observada, que se ha ido haciendo cada vez más frecuente ${ }^{77}$. En resumen, todo indica que aquí se ha expresado el concepto denominado "pendiente resbaladiza"78.

Adicionalmente, y en contraste con lo anterior, ha habido un progresivo ( $73 \%$ de aumento) y significativo rechazo de los médicos a la puesta en práctica de acciones destinadas a terminar con la vida de un paciente sin explícita solicitud ${ }^{79}$.

\section{Conclusión}

El desarrollo científico, técnico y organizacional de la medicina, así como la intromisión de consideraciones económicas y políticas en la lógica de la atención de salud, han hecho surgir nuevos requerimientos sobre la profesión médica. Las razones últimas de estas demandas son complejas 
y profundas. Algunas de ellas tienen que ver con una cierta incapacidad de la Medicina contemporánea de responder adecuadamente a las crecientes exigencias de los pacientes y de la sociedad. Con frecuencia, la misma sociedad es la que favorece la aparición de los problemas en razón de sus hábitos, costumbres y estilos de vida, sin asumir con plenitud la responsabilidad de enfrentarlos. También es patente que, en muchas áreas de la atención de salud, la adaptación de nuestra profesión a circunstancias nunca antes enfrentadas por la humanidad es todavía insuficiente, como es el cuidado de los ancianos, los desvalidos, los moribundos o el empleo de las nuevas tecnologías utilizadas en medicina. Buena parte de la insatisfacción colectiva con la medicina y la salud se vuelca sobre la profesión médica, exigiendo de ella una solución pronta a sus demandas. Uno de los requerimientos sociales, que algunos sectores solicitan y aún exigen de los profesionales de la salud, es la incorporación de la eutanasia en su práctica profesional.

Quienes promueven la eutanasia la justifican en razón de la autonomía de la persona: sosteniendo que cada cuál es libre de elegir la forma en que desea vivir o morir. Sin embargo, para otros, la autonomía personal, en principio deseable y aceptable, tiene límites, sin los cuales su ejercicio se transformaría en un grave problema social toda vez que hay bienes, como la vida, que poseen un valor jerárquicamente superior y anterior al de la autonomía.

El derecho a la vida es universalmente reconocido, de lo que deriva la prohibición que ésta sea quitada arbitrariamente por terceros, aún cuando se cuente con la anuencia del interesado. La experiencia de aquellos países que han legalizado la eutanasia muestra que esta práctica se extiende a situaciones en que ya no es el paciente quien la pide, sino que la hace la familia o los propios profesionales de la salud, al considerar ellos que el paciente está en una condición de vida no digna. También, se intenta fundamentar la eutanasia con la fórmula: "el derecho a vivir con dignidad", de controvertida interpretación. En rigor, esta expresión no ha de entenderse como una formulación de un derecho en el sentido preciso del ordenamiento jurídico sino, más bien, como una exigencia personal referida al universo ético. En definitiva, en la eutanasia está en juego el principio de respeto a la vida humana que se ha incorpo- rado al ethos universal, refrendado por diversas declaraciones de organismos internacionales que exhortan explícitamente a protegerla.

El examen objetivo de la naturaleza de la medicina, así como la tradición del arte médico, excluyen la práctica de la eutanasia. Ella no puede ser considerada un acto médico ni ser legítimamente solicitada a estos profesionales. Los médicos involucrados en la eutanasia, en lugar de proteger a los necesitados con los cuidados que pudieran brindarles, están realizando una práctica ajena al espíritu y tradición de la profesión, socavando gravemente las confianzas individuales y colectivas en la medicina, imprescindibles para una correcta y adecuada relación médico-paciente y de los pacientes con el sistema asistencial.

El avance de los conocimientos permite hoy día a la ciencia y arte médicos contar con recursos médicos cada vez más eficaces para combatir el dolor y otros sufrimientos de los pacientes y ofrecerles soluciones, como son los cuidados paliativos, cuyo desarrollo se visualiza como conveniente, ya que salvaguardan la dignidad de las personas y mantienen el espíritu de la profesión médica.

No hacerse cargo médica y humanamente de la agonía del enfermo, acelerándola o dando término intencionado a su vida para eliminar sufrimientos, costos económicos o responsabilidades políticas y sociales, vulnera el propósito de la profesión. Es posible que una generalización de la práctica de la eutanasia termine por afectar principalmente a quienes están más necesitados de cuidados -como los desvalidos, los ancianos, los enfermos crónicos y los moribundos- llegando incluso a dañar gravemente los derechos básicos de las personas.

Lo cierto es que, en muchos países, los debates médicos y jurídicos ya no versan tanto sobre el carácter lícito o ilícito de la eutanasia -el tema substantivo y de fondo- sino más bien sobre su mayor o menor conveniencia en casos concretos, sobre las normas que deberían regular su aplicación y sobre su mayor o menor aceptación social y política.

Corresponde a la Medicina y a quienes la practican identificar y llevar a cabo aquellos actos médicos que contribuyen, adecuada y eficazmente en este ámbito, a la ayuda de los pacientes, sean ellos terminales o no. La eutanasia no se encuentra entre aquellos.

Finalmente, además de las anteriores consideraciones, no se debe olvidar que, desde el 
punto de vista antropológico, la muerte no es un fenómeno mera y únicamente biológico, sino un evento personal esencial, con dimensiones éticas, culturales y religiosas.

\section{Referencias}

1. Grupo de Estudios de Ética Clínica de la Sociedad Médica de Santiago. El Enfermo Terminal. Rev Med Chile 2000; 128: 547-52.

2. Grupo de Estudios de Ética Clínica de la Sociedad Médica de Santiago. Sobre las acciones médicas proporcionadas y el uso de métodos extraordinarios de tratamiento. Rev Med Chile 2003; 131: 689-96.

3. Grupo de Estudios de Ética Clínica de la Sociedad Médica de Santiago. Diagnóstico de Muerte. Rev Med Chile 2004; 132: 95-107.

4. Grupo de Estudios de Ética Clínica de la Sociedad Médica de Santiago. La reanimación cardiorrespiratoria y la orden de no reanimar. Rev Med Chile 2007; 135: 669-79.

5. Gómez Rubí JA. Ética en medicina crítica. Triacastela, Madrid, 2002. pp. 228-9.

6. Pontificio Consejo para la Pastoral de los Agentes Sanitarios. Carta de los Agentes Sanitarios. \# 119. Ciudad del Vaticano, 1995.

7. Sgreccia, Elio. Manual de Bioética. Editorial Diana, México. 1996. p. 610.

8. Instituto Nacional de Estadísticas, Chile. Comisión Económica para América Latina y el Caribe. Chile: Proyecciones y Estimaciones de Población. Total País. 1950-2050. Publicación CEPAL OI No 208. Disponible en: http://www.ine.cl/canales/chile_estadistico/ demografia_y_vitales/proyecciones/Informes/Microsoft $\% 20$ Word\%20-\%20InforP_T.pdf [Consultado el 27 de diciembre de 2010].

9. Thomas, Evan. The case for killing granny. Newsweek, September 21, 2009. pp. 35-40.

10. Centro de Encuestas de La Tercera: "Eutanasia: 55\% de la población la aprueba, pero sólo $37 \%$ la aplicaría a un cercano”. Diario La Tercera, Martes 16 de mayo de 2006, p. 5

11. Biobarómetro. Universidad Católica de la Santísima Concepción. Segunda encuesta Actualidad. Agosto de 2006. Efectuada en mayores de 18 años, residentes en viviendas particulares del sector urbano de la comuna de Concepción. Muestreo por etapas, sorteo aleatorio de manzanas y luego sorteo aleatorio de viviendas, se entrevistó al jefe o jefa de hogar. 369 personas de un total de 53.658 viviendas. Error 5\%. Disponible en: http:// www.biobarometro.cl/encuestas/ii_encuesta_2006.pdf [Consultado el 27 de diciembre de 2010].

12. Esta petición le fue concedida, tras compleja transacción en la cual él se ofreció como víctima propiciatoria, satisfaciendo la furia de Zeus, que permitió que la muerte de Quirón tomara el lugar de la de Prometeo. C. Kerény. The heroes of the greeks. Thames and Hudson Ltd., London. 1959. Reprinted 1977. pp. 149-50.

13. “... Su muerte fue tranquila y como siempre la había deseado; porque cuando oía decir que había muerto alguno rápidamente y sin dolor, exponía al punto su deseo de morir él y todos los suyos de esta manera, lo que exponía con la palabra griega correspondiente..." Cayo Suetonio Tranquilo. Octavio Augusto, XCIX. En: Los Doce Césares. Editorial Iberia S.A., Barcelona 1962. pp. 110-1.

14. Instrucciones para el Dr. Brandt y el Reichsleiter Bouhler en Alemania, en septiembre de 1939. Cf. Henry Friedlander. The origins of Nazi Genocide: From Euthanasia to the Final Solution. University of North Carolina Press, Chapel Hill, NC. 1995. p.67

15. "Berlín, 1 de septiembre 1939. Reichsleiter Bouhlery Dr. med. Brandt están encargados, bajo su responsabilidad, de ampliar de tal manera las atribuciones de médicos a ser designados individualmente que se pueda conceder la muerte por gracia a los enfermos que bajo consideración más crítica y según la humana prevención son incurables. A. Hitler".

16. Real Academia Española. Diccionario de la Lengua Española. XXII Edición. Madrid. 2001.

17. Ciccone L. La ética y el término de la vida humana. En: Manual de Bioética General. Ed. A. Polaino-Lorente. $3^{\text {a }}$ Ed., RIALP, Madrid. 1997. pp. 423-38.

18. Sagrada Congregación para la Doctrina de la Fe, Declaración Iura et Bona, Roma, 5 de mayo de 1980. II. La Eutanasia.

19. Cordón López A. Eutanasia. Derecho a morir dignamente. Med Clin (Barc) 1995; 104: 92-3.

20. Ciccone L. Op.cit.

21. Gómez Rubí JA. Citado en 5. p. 253.

22. Onwuteaka-Philipsen BD, van der Heide A, Muller MT, Rurup M, Rietjens JA, Georges JJ, et al. Dutch experience of monitoring euthanasia. BMJ 2005; 331: 691-3.

23. Beca JP, Ortiz A, Solar S. Derecho a morir: un debate actual. Rev Med Chile 2005; 133: 601-6.

24. República de Chile. Código Penal, Título VIII.

25. Goic A. Apuntes sobre la eutanasia. Rev Med Chile 2005; 133: 371-5.

26. "Es lícito llevar a cabo una acción (u omitirla deliberadamente) incluso cuando esta elección comporte también un efecto malo, con las siguientes condiciones: 
1. Que la acción buscada sea en si buena, o por lo menos moralmente indiferente;

2. Que el efecto bueno sea directamente buscado por la persona que actúa, tanto por lo que se refiere a los efectos cuanto a la intención;

3. El permitir o aceptar indirectamente el efecto malo debe tener una motivación adecuada y proporcionada, lo cual quiere decir que el efecto bueno debe tener un efecto proporcionado para justificar la aceptación, aunque sea indirecta, del efecto malo." En Sgreccia E, op. cit. en 7, p. 167.

27. Quill TE. The Ambiguity of Clinical Intentions. N Engl J Med 1993; 329: 1039-40.

28. Quill TE, Dresser R, Borock DW. The Rule of Double Effect- A Critique of its Role in End-of-Life Decision Making. N Engl J Med 1997; 337: 1768-71.

29. Gómez-Lobo, A. Los Bienes Humanos. Ética de la ley natural. p. 109. Mediterráneo, Santiago. 2006.

30. Hardwig J. Is there a duty to die? Hastings Cent Rep 1997; 27: 34-42.

31. Grunfeld E, Coyle C, Whelan T, Clinch J, Reyno L, Earle CC, et al. Family caregiver burden: results of a longitudinal study of breast cancer patients and their principal caregivers. CMAJ 2004; 170: 1795-801.

32. Centers for Disease Control and Prevention and The Merck Company Foundation. The State of Aging and Health in America 2007. Whitehouse Station, NJ; The Merck Company Foundation; 2007.

33. Hentoff N. Euthanasia more "cost-effective" than care. The Lantern. January 2, 2001. Disponible en: http:// www.thelantern.com/2.1388/euthanasia-more-costeffective-than-care-1.100676 [Consultado el 15-feb2010].

34. Verhagen E, Sauer PJ. The Groningen Protocol-Euthanasia in Severely Ill Newborns. N Engl J Med 2005; 352: 959-62.

35. Rob de Jong TH. Deliberate termination of life of newborns with spina bifida, a critical reappraisal. Childs Nerv Syst 2008; 24: 13-28.

36. S.S. Juan Pablo II. Evangelium Vitae. Núm. 64-6.

37. Dorland's Illustrated Medical Dictionary. $30^{\text {th }}$. Ed. W.B. Saunders, Philadelphia, PA. 2003.

38. Webster's New World College Dictionary, $4^{\text {th }}$. Ed. Wiley Publishing Inc. Cleveland, OH. 2004.

39. Shorter Oxford English Dictionary. $5^{\text {th }}$. Ed. Oxford University Press, New York, 2002.

40. Le Petit Robert, Dictionnaire alphabétique et analogique de la langue française, Dictionnaires Le Robert (Paris) 1985.

41. Fields HL, Martin JB. Pain: Pathophysiology and Management. En: Harrison's Principles of Internal Medicine. $17^{\text {th }}$ Ed. Fauci AS, Braunwald E, Kasper L, et al. Eds. McGrawHill Medical, New York, 2008. pp. 81-7.

42. Ministerio de Salud de Chile. Guía Clínica Alivio del dolor por Cáncer Avanzado y Cuidados Paliativos.1st Ed. Santiago: Minsal, 2005.

43. Cherny NI, Portenoy RK. Sedation in the Management of refractory symptoms: guidelines for evaluation and treatment. J Palliat Care 1994; 10: 31-8.

44. Para la Sociedad Española de Cuidados Paliativos (SECPAL) se entiende por sedación paliativa la administración deliberada de fármacos, en las dosis y combinaciones requeridas, para reducir la conciencia de un paciente con una enfermedad avanzada o terminal, tanto como sea preciso para aliviar adecuadamente uno o más síntomas refractarios y con su consentimiento explícito, implícito o delegado. Disponible en: http://www.secpal. $\mathrm{com} /$ guiasm/index.php?acc $=$ see_guia\&id_guia $=8$ [Consultado el 27 de diciembre de 2010].

45. La SECPAL entiende por sedación terminal la administración deliberada de fármacos para lograr el alivio, inalcanzable con otras medidas, de un sufrimiento físico y/o psicológico, mediante la disminución suficientemente profunda y previsiblemente irreversible de la conciencia en un paciente cuya muerte se prevé muy próxima y con su consentimiento explícito, implícito o delegado. Disponible en: http://www.secpal.com/guiasm/index. php?acc $=$ see_guia\&id_guia $=8$ [Consultado el 27 de diciembre de 2010].

46. Cherny NI, Radbruch L, The Board of the European Association for Palliative Care. European Association for Palliative Care (EAPC) recommended framework for the use of sedation in palliative care. Palliative Medicine 2009; 23: 581-93.

47. Organización Mundial de la Salud. Comisión sobre Determinantes Sociales de la Salud. Resumen analítico del informe final. WHO/IER/CSDH/08.1. 2008.

48. Underbakke G, Zeller L. Putting the Diabetes Prevention Program into Practice: A program for Weight Loss and Cardiovascular Risk Reduction for Patients with Metabolic Syndrome or Type 2 Diabetes Mellitus. J Nutr Health Aging 2008;12: 745s-9s.

49. Saunders C. Hospice: a global network. J R Soc Med 2002; 95: 468.

50. Storey P. Goals of hospice care. Tex Med 1990; 86: 504.

51. Twycross R. Patient care: past, present and future. Omega (Westport) 2007-2008; 56: 7-19.

52. Marín LPP, Gac E, Homero, Hoyl M, Trinidad et al. Estudio comparativo de mujeres mayores de noventa años y ancianas menores institucionalizadas. Rev Med Chile 2004; 132: 33-9. 
53. Ferrater Mora J. Diccionario de Filosofía. Editorial Ariel S.A., Barcelona. 1999. p. 277.

54. Aumonier N, Beignier B, Letellier Ph. L'Euthanasie, PUF (Paris) 2001.

55. Aristóteles. Política. VII, 1335b. Ed. Gredos, Madrid. $1^{\text {a }}$. Ed. 2a . Reimpresión. 1999.

56. Reverte Coma JM. Las fronteras de la Medicina. Límites Éticos, Científicos y Jurídicos. Editorial Díaz de Santos, S.A., Madrid. 1983. pp. 101-15.

57. Juramento Hipocrático, traducción literal directa del original griego, según García Gual. En: Tratados Hipocráticos. Vol. I. Editorial Gredos, Madrid, 1983. pp. 77-83.

58. Herranz G. Los médicos y la eutanasia. OMC, 1998. Disponible en: http://www.unav.es/cdb/dhbgheutanasia. html [Consultado el 27 de diciembre de 2010].

59. Asociación Médica Mundial. Resolución sobre la eutanasia. 1987 y 2005. Disponible en: http://www.unav. es/cdb/ammmadrid4.html y en: http://www.wma.net/ en/30publications/10policies/e13/index.html [Consultado el 27 de diciembre de 2010].

60. Declaración Universal de los Derechos Humanos. 10 de diciembre de 1948. Disponible en: http://www.un.org/ es/documents/udhr/ [Consultado el 27 de diciembre de 2010].

61. Convención Americana sobre Derechos Humanos. Pacto de San José de Costa Rica. 22 de noviembre de 1969. Disponible en: http://www.acnur.org/biblioteca/ pdf/0001.pdf [Consultado el 27 de Diciembre de 2010].

62. Convenio para la Protección de los Derechos Humanos y de las Libertades Fundamentales. Roma, 4 de noviembre de 1950. Disponible en: http://www.acnur.org/biblioteca/pdf/1249.pdf [Consultado el 27 de diciembre de 2010].

63. Declaración Universal sobre Bioética y Derechos Humanos. UNESCO. 19 de octubre de 2005. Disponible en: http://unesdoc.unesco.org/images/0014/001461/ 146180S.pdf [Consultado el 27 de diciembre de 2010].

64. Van der Heide A, Onwuteaka-Philipsen BD, Rurup ML, Buiting HM, van Delden JJ, Hanssen-de Wolf JE, et al. End-of-life Practices in the Netherlands under the Euthanasia Act. N Engl J Med 2007; 356: 1957-65.
65. Vega Gutiérrez J. La práctica de la eutanasia en Bélgica y la "pendiente resbaladiza". Cuad Bioet 2007; 1: 71-87.

66. Colegio Médico de Chile A.G., Código de Ética. 2006.

67. República de Chile. Código Penal.

68. Goic A. El Fin de la Medicina. Publicaciones Técnicas Mediterráneo Ltda. Santiago de Chile, 2000. Págs. 20 y 38.

69. Hastings Center. Daniel Callahan (Director del proyecto). Los Fines de la Medicina. El establecimiento de unas prioridades nuevas. Traducción al español en: Cuadernos de la Fundació Víctor Grífols i Lucas. Los fines de la medicina - N.o 11 - (2004) Edita: Fundació Víctor Grífols i Lucas c/. Jesús i Maria, 6 - 08022 Barcelona.

70. Ibid. 69. p. 46

71. Ministerio de Salud. Aprueba normas de carácter técnico médico y administrativo para el cumplimiento de las garantías explícitas en salud de la ley 19.966. o 34. D.O. del 13.07.09. párrafo 3.4.

72. Ibid. 68. página 109.

73. Besio RM. Virtud artística, acto electivo e intuición creadora en la medicina. Memoria para optar al grado de Magister en Fundamentación Filosófica. Universidad de los Andes. Instituto de Filosofía. Santiago, Chile, 2007.

74. Mead M. The Cultural Shaping of the Ethical Situation. En: Who shall live? Medicine, Technology, Ethics. Kenneth Vaux, Ed. Fortress Press, Philadelphia, 1970. pp. 3-15.

75. Gómez-Lobo A. Citado en 29. p. 30.

76. Vega Gutiérrez J, Ortega I. La "pendiente resbaladiza" en la eutanasia en Holanda. Cuad Bioét 2007; 18: 89-104.

77. Sheldon T. Letting Him Down: making the euthanasia decision easier. BMJ 2006; 333: 556.

78. Se entiende por pendiente resbaladiza (slippery slope) o plano inclinado, el proceso por el cual, una vez admitido un determinado comportamiento cuestionable se hace más fácil la progresión hacia otro comportamiento situado en la misma línea que el primero, pero aun menos admisible o más cuestionable.

79. Onwuteaka-Philipsen BD, van der Heide A, Koper D, Keij-Deerenberg I, Rietjens JA, Rurup ML, et al. Euthanasia and other end-of-life decisions in the Netherlands in 1990, 1995 and 2001. Lancet 2003; 362 (9381): 395-9. 